\title{
$\perp$-order automorphisms of Hilbert space effect algebras: The two-dimensional case
}

\author{
Lajos Molnára) and Zsolt Páles ${ }^{\mathrm{b})}$ \\ Institute of Mathematics and Informatics, University of Debrecen, \\ 4010 Debrecen, P.O. Box 12, Hungary
}

(Received 10 November 2000; accepted for publication 20 December 2000)

It is well known that the $\perp$-order automorphisms of the effect algebra of a Hilbert space of dimension not less than three are implemented by unitary or antiunitary operators. The aim of this paper is to show that the same assertion also holds true in the two-dimensional case. (C) 2001 American Institute of Physics.

[DOI: 10.1063/1.1352052]

Let $H$ be a (real or complex) Hilbert space. The effect algebra of $H$ is the operator interval $[0, I]$ of all positive (self-adjoint, bounded linear) operators on $H$ which are bounded by the identity $I$. Effect algebras play a very important role in the mathematical foundations of quantum mechanics (see, e.g., Ref. 1). It is well known that if the dimension of $H$ is at least three, then the $\perp$-order automorphisms of $[0, I]$ (which are the bijective transformations of the effect algebra that preserve the order $\leqslant$ in both directions and also preserve the orthocomplementation $\perp: E \mapsto I$ $-E$ ) are implemented by unitary or antiunitary operators on $H$ (see Ref. 1, Sec. V 5 and Ref. 2). In fact, the proof is usually based on the fundamental theorem of projective geometry which holds true only in spaces of dimension not less than three. Because of the importance of effect algebras, it is a natural problem to clarify the situation in the two-dimensional case. In fact, Cassinelli et al. faced this issue in their paper ${ }^{2}$ (it is trivial that the corresponding assertion fails to hold in one dimension). Moreover, in their recent work, ${ }^{3}$ Lahti et al. showed that if the considered automorphism is induced via the functional calculus by a Borel function of the interval $[0,1]$, then it is necessarily the identity. The aim of this paper is to present the complete solution of the problem.

We have the following result.

Theorem: Let $H$ be a two-dimensional (real or complex) Hilbert space and let $[0, I]$ be the effect algebra of $H$. Let $\phi:[0, I] \rightarrow[0, I]$ be a bijective transformation with the property that

$$
E \leqslant F \Leftrightarrow \phi(E) \leqslant \phi(F) \text { and } \phi(I-E)=I-\phi(E)
$$

hold for every $E, F \in[0, I]$. Then there exists an either unitary or antiunitary operator $U$ on $H$ such that

$$
\phi(E)=U E U^{*} \quad(E \in[0, I])
$$

Proof: Several times in the proof we shall use the following easy observation: If $A, B$ are positive (self-adjoint) operators, $B \leqslant A$, and $A$ is of rank one, then $B=\lambda A$ for some scalar $\lambda$ $\geqslant 0$.

We recall that every bijection of the effect algebra of a Hilbert space which preserves the order in both directions necessarily preserves the projections as well as their ranks in both directions (see [Ref. 1, Theorem 5.8, p. 219]). Clearly, we have $\phi(0)=0, \phi(I)=I$.

In what follows, let $P$ be any rank-one projection on $H$ and let $Q$ be its orthogonal complement. Pick $\lambda \in[0,1]$. Since $0 \leqslant \phi(\lambda P) \leqslant \phi(P)$, it follows that $\phi(\lambda P)=f_{P}(\lambda) \phi(P)$ for some

\footnotetext{
${ }^{a)}$ Electronic mail: molnarl@math.klte.hu

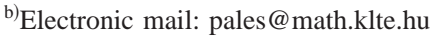


scalar $f_{P}(\lambda) \in[0,1]$. Clearly, $f_{P}:[0,1] \rightarrow[0,1]$ is a strictly monotone increasing bijection (observe that $\phi^{-1}$ has the same properties as $\left.\phi\right)$. If $\lambda \in[0,1]$, then from the inequality

$$
\phi(Q) \leqslant \phi(\lambda P+Q) \leqslant I=\phi(P)+\phi(Q)
$$

we obtain

$$
0 \leqslant \phi(\lambda P+Q)-\phi(Q) \leqslant \phi(P) .
$$

This implies that

$$
\phi(\lambda P+Q)=h_{P}(\lambda) \phi(P)+\phi(Q)
$$

for some scalar $h_{P}(\lambda) \in[0,1]$. Similiary to the case of $f_{P}, h_{P}:[0,1] \rightarrow[0,1]$ is a strictly monotone increasing bijection. We show that $h_{P}=f_{P}$. Indeed, since

$$
f_{P}(\lambda) \phi(P)=\phi(\lambda P) \leqslant \phi(\lambda P+Q)=h_{P}(\lambda) \phi(P)+\phi(Q),
$$

it follows that $f_{P} \leqslant h_{P}$. Considering the inverse $\phi^{-1}$ of $\phi$, it is easy to see that

$$
\phi^{-1}(\lambda \phi(P))=f_{P}^{-1}(\lambda) P
$$

and

$$
\phi^{-1}(\lambda \phi(P)+\phi(Q))=h_{P}^{-1}(\lambda) P+Q .
$$

Therefore, just as mentioned previously, we can deduce that $f_{P}^{-1} \leqslant h_{P}^{-1}$. Since the functions $f_{P}, h_{P}:[0,1] \rightarrow[0,1]$ are monotone increasing we then conclude that $f_{P}=h_{P}$. From the inequality

$$
\begin{aligned}
f_{P}(\lambda) \phi(P) & =\phi(\lambda P) \leqslant \phi(\lambda I)=\phi(\lambda P+\lambda Q) \leqslant \phi(\lambda P+Q) \\
& =h_{P}(\lambda) \phi(P)+\phi(Q)=f_{P}(\lambda) \phi(P)+\phi(Q)
\end{aligned}
$$

we infer that

$$
0 \leqslant \phi(\lambda I)-f_{P}(\lambda) \phi(P) \leqslant \phi(Q)
$$

and this implies that

$$
\phi(\lambda I)=f_{P}(\lambda) \phi(P)+k_{P}(\lambda) \phi(Q)
$$

for some scalar $k_{P}(\lambda) \in[0,1]$. Since $\phi(P), \phi(Q)$ run through the set of all pairs of mutually orthogonal rank-one projections, it now follows that $\phi(\lambda I)$ is diagonizable with respect to every basis. This gives us that $\phi(\lambda I)$ is a scalar operator, that is, $\phi(\lambda I)=f(\lambda) I$ for some scalar $f(\lambda)$ $\in[0,1]$. Clearly, $f:[0,1] \rightarrow[0,1]$ is a strictly monotone increasing bijection. Since

$$
f_{P}(\lambda) \phi(P)=\phi(\lambda P) \leqslant \phi(\lambda I)=f(\lambda) I \leqslant \phi(\lambda P+Q)=f_{P}(\lambda) \phi(P)+\phi(Q),
$$

it follows that $f=f_{P}=h_{P}$. So, we have

$$
\phi(\lambda P)=f(\lambda) \phi(P)
$$

and

$$
\phi(\lambda P+Q)=f(\lambda) \phi(P)+\phi(Q) .
$$

The argument leading to this conclusion can also be applied to get 


$$
\phi(\lambda Q)=f(\lambda) \phi(Q)
$$

and

$$
\phi(P+\lambda Q)=\phi(P)+f(\lambda) \phi(Q) \text {. }
$$

For any $\lambda, \mu \in[0,1]$ we have

$$
f(\lambda) \phi(P)=\phi(\lambda P) \leqslant \phi(\lambda P+\mu Q) \leqslant \phi(\lambda P+Q)=f(\lambda) \phi(P)+\phi(Q)
$$

and

$$
f(\mu) \phi(Q)=\phi(\mu Q) \leqslant \phi(\lambda P+\mu Q) \leqslant \phi(P+\mu Q)=\phi(P)+f(\mu) \phi(Q) .
$$

From the first inequality we infer that

$$
\phi(\lambda P+\mu Q)=f(\lambda) \phi(P)+\alpha \phi(Q)
$$

for some $\alpha \in[0,1]$, while from the second one we have

$$
\phi(\lambda P+\mu Q)=\beta \phi(P)+f(\mu) \phi(Q)
$$

for some $\beta \in[0,1]$. Comparing these equalities we get

$$
\phi(\lambda P+\mu Q)=f(\lambda) \phi(P)+f(\mu) \phi(Q)
$$

for every $\lambda, \mu \in[0,1]$.

Observe that $f$ has the following symmetry property: $f(\lambda)+f(1-\lambda)=1$. Indeed, this follows from the equality $\phi(\lambda I)+\phi((1-\lambda) I)=I$.

Our next claim is to obtain a functional equation for $f$. In order to do this, we recall the following notation from Ref. 4 . If $E$ is an effect on a Hilbert space and $\varphi$ is a unit vector, then let

$$
\lambda\left(E, P_{\varphi}\right)=\sup \left\{\lambda \in[0,1]: \lambda P_{\varphi} \leqslant E\right\},
$$

where $P_{\varphi}$ is the orthogonal projection onto the subspace generated by $\varphi$. Suppose that $E$ $\in[0, I]$. Then (Ref. 4, Theorem 4) tells us that

$$
\lambda\left(E, P_{\varphi}\right)=\left\{\begin{array}{l}
\left\|E^{-1 / 2} \varphi\right\|^{-2}, \quad \text { if } \varphi \in \operatorname{ran}\left(E^{1 / 2}\right) \\
0, \quad \text { else }
\end{array} .\right.
$$

Fix mutually orthogonal rank-one projections $P, Q$ on $H$. Pick $\mu \in] 0,1[$ and let $E=\mu P+Q$. Take any rank-one projection $R$ on $H$ which is neither equal to nor orthogonal to $P$. Similarly to the second step of the proof (Ref. 2, Lemma 3), it is easy to verify that

$$
\lambda(E, R)=\frac{\mu}{\mu+(1-\mu) \operatorname{tr} P R} .
$$

Indeed, if $r$ is a unit vector in the range of $R$, then using the above-mentioned result from Ref. 4, we compute

$$
\begin{aligned}
\lambda(E, R) & =\|((1 / \sqrt{\mu}) P+Q) r\|^{-2}=\frac{1}{(1 / \mu)\|P r\|^{2}+\|Q r\|^{2}}=\frac{\mu}{\|P r\|^{2}+\mu\|Q r\|^{2}} \\
& =\frac{\mu}{\mu\|P r\|^{2}+\mu\|Q r\|^{2}+(1-\mu)\|P r\|^{2}}=\frac{\mu}{\mu+(1-\mu)\|P r\|^{2}}=\frac{\mu}{\mu+(1-\mu) \operatorname{tr} P R} .
\end{aligned}
$$

By the definition of $\lambda(E, R)$, it is clear that 


$$
\begin{aligned}
f(\lambda(E, R)) & =\sup \{f(\lambda): \lambda R \leqslant E\}=\sup \{f(\lambda): \phi(\lambda R) \leqslant \phi(E)\} \\
& =\sup \{f(\lambda): f(\lambda) \phi(R) \leqslant \phi(E)\}=\lambda(\phi(E), \phi(R)) .
\end{aligned}
$$

Since $\phi(E)=\phi(\mu P+Q)=f(\mu) \phi(P)+\phi(Q)$, it follows that

$$
f\left(\frac{\mu}{\mu+(1-\mu) \operatorname{tr} P R}\right)=\frac{f(\mu)}{f(\mu)+(1-f(\mu)) \operatorname{tr} \phi(P) \phi(R)} .
$$

As the quantities $\operatorname{tr} P R$ and $\operatorname{tr} \phi(P) \phi(R)$ do not depend on $\mu$, it follows from this equality that $\operatorname{tr} \phi(P) \phi(R)$ can be uniquely expressed as a function of $\operatorname{tr} P R$. Denoting $g(\operatorname{tr} P R)$ $=\operatorname{tr} \phi(P) \phi(R)$, we get a bijective function $g:] 0,1[\rightarrow] 0,1[$ for which

$$
f\left(\frac{\mu}{\mu+(1-\mu) \nu}\right)=\frac{f(\mu)}{f(\mu)+(1-f(\mu)) g(\nu)} \quad(\mu, \nu \in] 0,1[)
$$

holds true. Replacing $\mu$ by $1-\mu$ and $\nu$ by $1-\nu$ we have the following more aesthetic equality:

$$
f\left(\frac{1-\mu}{1-\mu \nu}\right)=\frac{1-f(\mu)}{1-f(\mu) g(\nu)} \quad(\mu, \nu \in] 0,1[) .
$$

Here we have used the fact that $g$ has the same symmetry property as $f$. This follows from the following equality:

$$
\begin{aligned}
g(1-\operatorname{tr} P R)= & g(\operatorname{tr} P-\operatorname{tr} P R)=g(\operatorname{tr}(P(I-R)))=\operatorname{tr} \phi(P) \phi(I-R)=\operatorname{tr} \phi(P)(I-\phi(R))=\operatorname{tr} \phi(P) \\
& -\operatorname{tr} \phi(P) \phi(R)=1-g(\operatorname{tr} P R) .
\end{aligned}
$$

Now, we turn to the solution of the functional equation (2). Our corresponding result is formulated in the following separate statement.

Proposition: Let $f, g:] 0,1[\rightarrow] 0,1[$ be functions. Suppose that $f$ is a strictly monotone increasing bijection and that $f(1-x)=1-f(x)$ and $g(1-y)=1-g(y)$ hold for every $x, y \in] 0,1[$. If

$$
f\left(\frac{1-x}{1-x y}\right)=\frac{1-f(x)}{1-f(x) g(y)} \quad(x, y \in] 0,1[),
$$

then $f$ and $g$ are the identities on $] 0,1[$.

Proof: The function $f$ being continuous, Eq. (3) implies the continuity of $g$.

Observe that, with the notation

$$
\begin{gathered}
\alpha(t):=\frac{1}{1+e^{t}} \quad(t \in \mathbb{R}), \\
\beta(x):=\ln \frac{x}{1-x} \quad(x \in] 0,1[), \\
\gamma(y):=\ln (1-y) \quad(y \in] 0,1[),
\end{gathered}
$$

we have the following identity:

$$
\frac{1-x}{1-x y}=\frac{1}{1+\exp \left(\ln \frac{x}{1-x}+\ln (1-y)\right)}=\alpha(\beta(x)+\gamma(y))
$$

for all $x, y \in] 0,1[$. Therefore, Eq. (3) can be rewritten as 


$$
f \circ \alpha(\beta(x)+\gamma(y))=\alpha(\beta \circ f(x)+\gamma \circ g(y)) \quad(x, y \in] 0,1[) .
$$

Substituting $x=\beta^{-1}(u)$ and $y=\gamma^{-1}(v)$ into Eq. (4) and applying the inverse function of $\alpha$ to both sides of Eq. (4), we get

$$
\alpha^{-1} \circ f \circ \alpha(u+v)=\beta \circ f \circ \beta^{-1}(u)+\gamma \circ g \circ \gamma^{-1}(v)
$$

for all $u \in \mathbb{R}$ and $v \in]-\infty, 0[$. Thus the functions

$$
F:=\alpha^{-1} \circ f \circ \alpha, \quad G:=\beta \circ f \circ \beta^{-1}, \quad H:=\gamma \circ g \circ \gamma^{-1}
$$

satisfy the following so-called Pexider equation:

$$
F(u+v)=G(u)+H(v) \quad(u \in \mathbb{R}, v \in]-\infty, 0[) .
$$

Then, by known results of the theory of functional equations (cf. Ref. 5 or 6 ) and by the continuity of $F, G, H$, it follows that there exist constants $a, b, c \in \mathbb{R}$ such that

$$
\begin{gathered}
F(w)=c w+a+b \quad(w \in \mathbb{R}), \\
G(u)=c u+a \quad(u \in \mathbb{R}), \\
H(v)=c v+b \quad(v \in]-\infty, 0[) .
\end{gathered}
$$

Using Eq. (6) and the definition of $G$, we get that $\beta \circ f(x)=c \beta(x)+a$, whence

$$
f(x)=\frac{x^{c}}{x^{c}+e^{-a}(1-x)^{c}} \quad(x \in] 0,1[) .
$$

Similarly, the definitions of $H$ and $\gamma$, and Eq. (7) yield

$$
g(y)=1-e^{b}(1-y)^{c} \quad(y \in] 0,1[) .
$$

The function $f$ being strictly increasing, $G$ is also increasing whence we get that $c>0$. Thus $f$ satisfies the identity $f(1-x)=1-f(x)$ if and only if $a=0$. The analogous identity for $g$ is valid if and only if $b=0, c=1$. Therefore $f(x)=g(x)=x$ for all $x \in] 0,1[$.

Returning to the proof of our theorem, since the above-mentioned function $g$ is the identity, we have $\operatorname{tr} P Q=\operatorname{tr} \phi(P) \phi(Q)$ for any rank-one projections $P, Q$ on $H$. Hence, using Wigner's theorem on symmetry transformations we obtain that there exists an either unitary or antiunitary operator $U$ on $H$ such that

$$
\phi(P)=U P U^{*}
$$

for every rank-one projection $P$ on $H$. As $f$ is also the identity, from Eq. (1) we infer that

$$
\phi(\lambda P+\mu Q)=\lambda \phi(P)+\mu \phi(Q)=\lambda U P U^{*}+\mu U Q U^{*}=U(\lambda P+\mu Q) U^{*},
$$

which means that $\phi(E)=U E U^{*}$ holds for every effect $E$ on $H$.

This completes the proof of our theorem.

We are very grateful to Professor S. Pulmannová for drawing our attention to the problem and Professor P. Lahti for informing us about the origin of the problem treated in the paper. This research was supported by the following sources: Hungarian National Foundation for Scientific Research (OTKA), Grant Nos. T-030082, T-031995 and the Ministry of Education, Hungary, Reg. Nos. FKFP 0310/1997, 0349/2000. 
${ }^{1}$ G. Ludwig, Foundations of Quantum Mechanics (Springer, Berlin, 1983), Vol. I.

${ }^{2}$ G. Cassinelli, E. De Vito, P. Lahti, and A. Levrero, "A theorem of Ludwig revisited," Found. Phys. 30, 1755-1761 (2000).

${ }^{3}$ P. Lahti, M. Mączynski and K. Ylinen, "A note on order and orthocomplementation preserving automorphisms of the set of effect operators on a Hilbert space," Lett. Math. Phys. (submitted).

${ }^{4}$ P. Busch and S. P. Gudder, “Effects as functions on projective Hilbert spaces,' Lett. Math. Phys. 47, 329-337 (1999).

${ }^{5}$ J. Aczél, Lectures on Functional Equations and their Applications (Academic, New York, 1966).

${ }^{6} \mathrm{M}$. Kuczma, An Introduction to the Theory of Functional Equations and Inequalities (Państwowe Wydawnictwo Naukowe, Warszawa, 1985). 